\title{
Existence of Nontrivial Solutions for Unilaterally Asymptotically Linear Three-Point Boundary Value Problems
}

\author{
Hongyu Li ${ }^{1,2}$ \\ ${ }^{1}$ Department of Mathematics, Shandong University of Science and Technology, Qingdao, Shandong 266590, China \\ ${ }^{2}$ Mathematics and Science College, Shanghai Normal University, Shanghai 200234, China \\ Correspondence should be addressed to Hongyu Li; sdlhy1978@163.com
}

Received 5 February 2014; Accepted 24 April 2014; Published 11 May 2014

Academic Editor: Paul W. Eloe

Copyright (c) 2014 Hongyu Li. This is an open access article distributed under the Creative Commons Attribution License, which permits unrestricted use, distribution, and reproduction in any medium, provided the original work is properly cited.

Using fixed point theorems in ordered Banach spaces with the lattice structure, we consider the existence of nontrivial solutions under the condition that the nonlinear term can change sign and study the existence of sign-changing solutions for some second order three-point boundary value problems. Our results improve and generalize on those in the literatures.

\section{Introduction}

In this paper, we shall discuss the existence of nontrivial solutions for the following boundary value problem:

$$
\begin{gathered}
-u^{\prime \prime}(t)=g(t, u(t)), \quad 0 \leq t \leq 1, \\
u(0)=0, \quad u(1)=\alpha u(\beta),
\end{gathered}
$$

where $g:[0,1] \times(-\infty,+\infty) \rightarrow(-\infty,+\infty)$ is continuous, $0<\alpha<1,0<\beta<1$.

Many problems of different areas of physics and applied mathematics can be changed into multipoint boundary value problems for ordinary differential equations (see [1]). In [2], Gupta firstly studied three-point boundary value problems for nonlinear second order ordinary differential equations in 1992. Since then, many authors have been concerned with second order multipoint boundary value problems (see [320] and references therein). For example, some authors have studied the existence and multiplicity of positive solutions for nonlinear multipoint boundary value problems under the condition that the nonlinear term may be nonnegative by applying Krasnosel'skii's fixed point theorem, theory of fixed point index, and so on (see [3-8]). Meanwhile, some authors considered the existence of nontrivial solutions when the nonlinear term can be negative; for example, see [911] and references therein. For instance, in [10], under the assumption of non-well-ordered upper and lower solutions, some multiplicity results for solutions of three-point boundary value problems (1) have been obtained using the fixed point index theory. On the other hand, some authors have considered the existence of sign-changing solutions to some boundary value problems (see $[12-16,18]$ and references therein). For example, in [13], by using the fixed point index method, $\mathrm{Xu}$ and Sun have considered the existence of signchanging solutions for the following three-point boundary value problem:

$$
\begin{gathered}
y^{\prime \prime}(t)+g(y(t))=0, \quad 0 \leq t \leq 1, \\
y(0)=0, \quad y(1)=\alpha y(\beta) .
\end{gathered}
$$

In [18], Rynne has considered the following second order $m$-point boundary value problem:

$$
\begin{gathered}
-u^{\prime \prime}=f(u), \\
u(0)=0, \quad u(1)=\sum_{i=1}^{m-2} \alpha_{i} u\left(\eta_{i}\right),
\end{gathered}
$$

where $f:(-\infty,+\infty) \rightarrow(-\infty,+\infty)$ is continuous. The author has used global bifurcation theorem to obtain signchanging solutions of the boundary value problem (3) under some conditions on the asymptotic behavior of $f$. 
Inspired by [9-18, 21-25], we shall use fixed point theorems derived by Liu and Sun [21] to consider the existence of nontrivial solutions for BVP (1). There are two main features. Firstly, the used methods are fixed point theorems with respect to noncone mappings, which are different from those of [9-18]. Secondly, when we consider the existence of signchanging solution to BVP (1), we generalize the nonlinear term $g(t, \varphi)$, which is different from $[13-15,18]$.

The organization of this paper is as follows. In Section 2, some preliminaries and lemmas are given including some properties of the lattice and some lemmas that will be used to prove the main results. In Section 3, we shall give the main results. Finally, in Section 4, concrete examples are given to illustrate applications of obtained main results.

\section{Preliminaries and Some Lemmas}

Let $E$ be an ordered Banach space in which the partial ordering $\leq$ is induced by a cone $P \subseteq E$. $P$ is called normal if there exists $N>0$ such that $\theta \leq x \leq y$ implies $\|x\| \leq N\|y\|$ (see [26]).

Definition 1 (see $[16,21-23])$. We call $E$ a lattice under the partial ordering $\leq$, if $\sup \{x, y\}$ and $\inf \{x, y\}$ exist for arbitrary $x, y \in E$.

For $x \in E$, let

$$
x^{+}=\sup \{x, \theta\}, \quad x^{-}=\sup \{-x, \theta\},
$$

$x^{+}$and $x^{-}$are called the positive part and the negative part of $x$ respectively, and clearly $x=x^{+}-x^{-}$. Take $|x|=x^{+}+x^{-}$, then $|x| \in P$, and $|x|$ is called the module of $x$. For convenience, we use the notations $x_{+}=x^{+}, x_{-}=-x^{-}$, and obviously $x_{+} \in P$, $x_{-} \in(-P), x=x_{+}+x_{-}($see $[16,21-23])$.

Definition 2 (see $[16,21-23]$ ). Let $D \subset E$ and $A: D \rightarrow E$ be a nonlinear operator. $A$ is said to be quasi-additive on lattice, if there exists $v^{*} \in E$ such that

$$
A x=A x_{+}+A x_{-}+v^{*}, \quad \forall x \in D .
$$

Definition 3 (see [21]). Let $E$ be a Banach space with a cone $P$ and let $A: E \rightarrow E$ be a nonlinear operator. We say that $A$ is a unilaterally asymptotically linear operator along $P$, if there exists a bounded linear operator $L$ such that

$$
\lim _{x \in P,\|x\| \rightarrow \infty} \frac{\|A x-L x\|}{\|x\|}=0,
$$

where $L$ is said to be the derived operator of $A$ along $P$ and will be denoted by $A_{P}^{\prime}$.

Remark 4. The operator $A$ in Definition 3 is not assumed to be a cone mapping.

Let $P$ be a cone of Banach space $E$. $x$ is said to be a positive fixed point of $A$ if $x \in(P \backslash\{\theta\})$ is a fixed point of $A$; $x$ is said to be a negative fixed point of $A$ if $x \in((-P) \backslash\{\theta\})$ is a fixed point of $A ; x$ is said to be a sign-changing fixed point of $A$ if $x \notin(P \cup(-P))$ is a fixed point of $A$ (see [21-23]).
Lemma 5 (see [21]). Let $E$ be a Banach space with a lattice structure, let $P$ be a normal cone of $E$, and let $A: E \rightarrow E$ be completely continuous and quasi-additive on lattice. Suppose that there exist $\nu_{1}, \nu_{2} \in P$ and a positive bounded linear operator $L: E \rightarrow E$ with $r(L)<1$, such that

$$
\begin{gathered}
A x \geq-v_{1}, \quad \forall x \in P ; \\
A x \geq L x-v_{2}, \quad \forall x \in(-P) .
\end{gathered}
$$

In addition, assume that $A \theta=\theta$; the Fréchet derivative $A_{\theta}^{\prime}$ of $A$ at $\theta$ exists, 1 is not an eigenvalue of $A_{\theta}^{\prime}$, the sum of the algebraic multiplicities for all eigenvalues of $A_{\theta}^{\prime}$, lying in the interval $(1, \infty)$, is an odd number, and $A_{P}^{\prime}$ exists, $r\left(A_{P}^{\prime}\right)<1$.

Then $A$ has at least one nontrivial fixed point.

Lemma 6 (see [21]). Let E, P, A, and $L$ be as in Lemma 5. Suppose that there exist $\mu_{1}, \mu_{2} \in P$ such that

$$
\begin{aligned}
& A x \leq \mu_{1}, \quad \forall x \in(-P) \\
& A x \leq L x+\mu_{2}, \quad \forall x \in P .
\end{aligned}
$$

In addition, assume that $A \theta=\theta$; the Fréchet derivative $A_{\theta}^{\prime}$ of $A$ at $\theta$ exists; 1 is not an eigenvalue of $A_{\theta}^{\prime}$, and $A_{(-P)}^{\prime}$ exists; $r\left(A_{(-P)}^{\prime}\right)>1$ and 1 is not an eigenvalue of $A_{(-P)}^{\prime}$ corresponding a positive eigenvector.

Then A has at least one nontrivial fixed point.

Lemma 7 (see [21]). Suppose that $E$ is an ordered Banach space with a lattice structure, $P$ is a normal cone of $E$, and $A$ is quasi-additive on the lattice. Assume that

(i) $A$ is strongly increasing on $P$ and $-P$;

(ii) both $A_{P}^{\prime}$ and $A_{(-P)}^{\prime}$ exist with $r\left(A_{P}^{\prime}\right)>1$ and $r\left(A_{(-P)}^{\prime}\right)>1$, and 1 is not an eigenvalue of $A_{P}^{\prime}$ or $A_{(-P)}^{\prime}$ corresponding a positive eigenvector;

(iii) $A \theta=\theta$; the Fréchet derivative $A_{\theta}^{\prime}$ of $A$ at $\theta$ is strongly positive and $r\left(A_{\theta}^{\prime}\right)<1$;

(iv) the Fréchet derivative $A_{\infty}^{\prime}$ of $A$ at $\infty$ exists; 1 is not an eigenvalue of $A_{\infty}^{\prime}$; the sum of the algebraic multiplicities for all eigenvalues of $A_{\infty}^{\prime}$, lying in the interval $(1, \infty)$, is an even number.

Then $A$ has at least three nontrivial fixed points containing one sign-changing fixed point.

Let $E=C[0,1]$. Define the norm $\|u\|=\max _{0 \leq t \leq 1}|u(t)|$; then $E$ is an ordered Banach space. It is obvious that $P=\{u \in$ $E \mid u(t) \geq 0, t \in[0,1]\}$ is a normal cone of $E$ and that $E$ is a lattice under the partial order $\leq$ which is induced by $P$ (see $[16,21-23])$.

For convenience, we list the following conditions.

$\left(\mathrm{H}_{1}\right)$ The sequence of positive solutions of the equation

$$
\sin \sqrt{x}=\alpha \sin \beta \sqrt{x}
$$

is

$$
0<\lambda_{1}<\lambda_{2}<\cdots<\lambda_{n}<\lambda_{n+1}<\cdots
$$


$\left(\mathrm{H}_{2}\right) \lim _{\varphi \rightarrow+\infty}(g(t, \varphi) / \varphi)=\xi$ uniformly on $[0,1]$.

$\left(\mathrm{H}_{3}\right) \lim _{\varphi \rightarrow-\infty}(g(t, \varphi) / \varphi)=\rho$ uniformly on $[0,1]$.

$\left(\mathrm{H}_{4}\right) g(t, 0) \equiv 0, \lim _{\varphi \rightarrow 0}(g(t, \varphi) / \varphi)=\eta$ uniformly on $[0,1]$.

By [4], it is well known that BVP (1) can be converted to the following nonlinear Hammerstein equation:

$$
u(t)=\int_{0}^{1} Z(t, s) g(s, u(s)) d s, \quad t \in[0,1]
$$

where

$$
\begin{gathered}
Z(t, s)=k(t, s)+\frac{\alpha t}{1-\alpha \beta} k(\beta, s), \\
k(t, s)= \begin{cases}t(1-s), & 0 \leq t \leq s \leq 1, \\
s(1-t), & 0 \leq s \leq t \leq 1 .\end{cases}
\end{gathered}
$$

Define the operators

$$
\begin{gathered}
(\Phi u)(t)=\int_{0}^{1} Z(t, s) g(s, u(s)) d s, \quad t \in[0,1], \\
(L u)(t)=\int_{0}^{1} Z(t, s) u(s) d s, \quad t \in[0,1], \\
(G u)(t)=g(t, u(t)), \quad t \in[0,1],
\end{gathered}
$$

where $Z(t, s)$ is defined by (12), and obviously $\Phi=L G$. It is obvious that fixed points of $\Phi$ are solutions of BVP (1) (see [4]).

Lemma 8 (see [13]). Let $\delta$ be a positive number. The eigenvalues of the linear operator $\delta L$ are

$$
\frac{\delta}{\lambda_{1}}, \frac{\delta}{\lambda_{2}}, \ldots, \frac{\delta}{\lambda_{n}}, \ldots,
$$

and the algebraic multiplicity of each positive eigenvalue $\delta / \lambda_{n}$ of the linear operator $\delta L$ is equal to 1 , where $\lambda_{n}$ is defined by (10).

Lemma 9. Let $\Phi$ and L be defined as (14) and (15), respectively. Then

(i) $\Phi, L: E \rightarrow$ E are completely continuous;

(ii) $\Phi$ is quasi-additive on the lattice;

(iii) $r(L)=1 / \lambda_{1}$, where $r(L)$ is the spectral radius of the operator $L$.

Proof. By [4], we know that (i) holds. The proof of (ii) is similar to that of $[16,21-25]$, so we omit it. We easily know that

$$
r(L)=\sup _{\lambda \in\left\{1 / \lambda_{n}, n=1,2, \ldots\right\}}|\lambda|=\frac{1}{\lambda_{1}} .
$$

Lemma 10. Let $\Phi$ and $L$ be defined as (14) and (15), respectively. Then (i) if $\left(\mathrm{H}_{2}\right)$ holds, then $\Phi_{P}^{\prime}=\xi L$;

(ii) if $\left(H_{3}\right)$ holds, then $\Phi_{(-P)}^{\prime}=\rho L$;

(iii) if $\left(H_{4}\right)$ holds, then $\Phi_{\theta}^{\prime}=\eta L$.

Proof. (i) By $\left(\mathrm{H}_{2}\right)$, for any $\epsilon>0$, there exists $R>0$ such that

$$
|g(t, \varphi)-\xi \varphi| \leq \epsilon \varphi, \quad \forall t \in[0,1], \varphi>R .
$$

Let $M_{R}=\max _{t \in[0,1], 0 \leq \varphi \leq R}|g(t, \varphi)|$. Then

$|(G u)(t)-\xi u(t)|=|g(t, u(t))-\xi u(t)| \leq M_{R}+\xi R+\epsilon\|u\|$,

$\forall u \in P, \quad\|u\| \geq R$,

and hence

$$
\|\Phi u-\xi L u\|=\|L(G u)-\xi L u\| \leq\|L\|\left(M_{R}+\xi R+\epsilon\|u\|\right),
$$

which means

$$
\liminf _{u \in P,\|u\| \rightarrow \infty} \frac{\|\Phi u-\xi L u\|}{\|u\|} \leq \epsilon\|L\|
$$

that is,

$$
\lim _{u \in P,\|u\| \rightarrow \infty} \frac{\|\Phi u-\xi L u\|}{\|u\|}=0,
$$

so by Definition 3, we have $\Phi_{P}^{\prime}=\xi L$.

(ii) Similar to the proof of (i), we can prove that conclusion (ii) holds.

(iii) By $\left(\mathrm{H}_{4}\right)$, since $g(t, 0) \equiv 0, \forall t \in[0,1], \Phi \theta=\theta$. And for any $\epsilon>0$, there exists $r>0$ such that

$$
|g(t, \varphi)-\eta \varphi| \leq \epsilon|\varphi|, \quad \forall t \in[0,1],|\varphi| \leq r .
$$

Then

$$
\begin{array}{r}
|(G u)(t)-\eta u(t)|=|g(t, u(t))-\eta u(t)| \leq \epsilon\|u\|, \\
\forall\|u\| \leq r,
\end{array}
$$

and hence

$$
\begin{array}{r}
\|\Phi u-\Phi \theta-\eta L u\|=\|L(G u)-\eta L u\| \leq\|L\| \epsilon\|u\|, \\
\forall\|u\| \leq r ;
\end{array}
$$

that is,

$$
\lim _{\|u\| \rightarrow 0} \frac{\|\Phi u-\Phi \theta-\eta L u\|}{\|u\|}=0,
$$

which means $\Phi_{\theta}^{\prime}=\eta L$.

\section{Main Results}

Theorem 11. Assume that $\left(H_{1}\right)-\left(H_{4}\right)$ hold. In addition, suppose that 
(i) $0<\xi<\lambda_{1}, 0<\rho<\lambda_{1}$;

(ii) there exists an odd number $n>0$ such that

$$
\lambda_{n}<\eta<\lambda_{n+1}
$$

where $\lambda_{1}, \lambda_{n}$, and $\lambda_{n+1}$ are defined by (10).

Then BVP (1) has at least one nontrivial solution.

Proof. By $\left(\mathrm{H}_{2}\right)$ and $\xi>0$, there exists $c>0$ such that

$$
g(t, \varphi) \geq-c, \quad \forall t \in[0,1], \varphi \geq 0
$$

and hence by (14) and (15), we have

$$
(\Phi u)(t)=(L G u)(t) \geq-L c, \quad \forall t \in[0,1], u \in P .
$$

And we easily know that $L c \in P$. By $0<\rho<\lambda_{1}$, we know that there exists $d>0$ such that

$$
0<\rho+d<\lambda_{1}
$$

By $\left(\mathrm{H}_{3}\right)$ and (31), there exists $\mathrm{C}>0$ such that

$$
g(t, \varphi) \geq(\rho+d) \varphi-C, \quad \forall t \in[0,1], \varphi \leq 0,
$$

and therefore by (14) and (15), we get

$$
(\Phi u)(t) \geq(\rho+d)(L u)(t)-L C, \quad \forall t \in[0,1], u \in(-P) .
$$

And we easily know that $L C \in P$. By (30) and (33), we know that (7) of Lemma 5 is satisfied.

Set $\widetilde{L}=(\rho+d) L$; by (31) and Lemma 9, we have $r(\widetilde{L})=$ $(\rho+d) r(L)<\lambda_{1} r(L)=1$.

By Lemma 10, we have $\Phi_{P}^{\prime}=\xi L, \Phi_{\theta}^{\prime}=\eta L$.

By $\left(\mathrm{H}_{4}\right)$, we easily know that $\Phi \theta=\theta$. By Lemma 8 , we know that $\eta / \lambda_{1}, \eta / \lambda_{2}, \ldots, \eta / \lambda_{n}, \ldots$ are the eigenvalues of $\Phi_{\theta}^{\prime}$.

Since $\lambda_{n}<\eta<\lambda_{n+1}$ and $n>0$ is an odd number, 1 is not the eigenvalue of $\Phi_{\theta}^{\prime}$, and the sum of the algebraic multiplicities for all eigenvalues of $\Phi_{\theta}^{\prime}$, lying in the interval $(1, \infty)$, is an odd number.

By $0<\xi<\lambda_{1}$ and Lemma 9, $r\left(\Phi_{P}^{\prime}\right)=\xi L=\xi r(L)<$ $\lambda_{1} r(L)=1$.

So the conditions of Lemma 5 are satisfied. Lemma 5 assures that $\Phi$ has at least one nontrivial fixed point. So BVP (1) has at least one nontrivial solution.

Theorem 12. Assume that $\left(H_{1}\right)-\left(H_{4}\right)$ hold. In addition, suppose that

(i) $0<\xi<\lambda_{1}$;

(ii) there exist natural numbers $n_{1} \geq 1$ and $n_{2} \geq 1$ such that

$$
\lambda_{n_{1}}<\rho<\lambda_{n_{1}+1}, \quad \lambda_{n_{2}}<\eta<\lambda_{n_{2}+1},
$$

where $\lambda_{1}, \lambda_{n_{1}}, \lambda_{n_{1}+1}, \lambda_{n_{2}}, \lambda_{n_{2}+1}$ are defined by (10).

Then BVP (1) has at least one nontrivial solution.
Proof. By $\left(\mathrm{H}_{4}\right)$, we easily know that $\Phi \theta=\theta$. By Lemma 10, we have $\Phi_{P}^{\prime}=\xi L, \Phi_{(-P)}^{\prime}=\rho L, \Phi_{\theta}^{\prime}=\eta L$.

From Lemma $8, \eta / \lambda_{1}, \eta / \lambda_{2}, \ldots, \eta / \lambda_{n}, \ldots$ are the eigenvalues of the linear operator $\Phi_{\theta}^{\prime}$. Since $\lambda_{n_{2}}<\eta<\lambda_{n_{2}+1}, 1$ is not an eigenvalues of $\Phi_{\theta}^{\prime}$. Since $\lambda_{n_{1}}<\rho<\lambda_{n_{1}+1}$ and (10), we know that $\rho>\lambda_{1}>0$. So $r\left(\Phi_{(-P)}^{\prime}\right)=\rho r(L)=\rho / \lambda_{1}>1$.

By $\left(\mathrm{H}_{3}\right)$ and $\rho>0$, there exists $m>0$ such that

$$
g(t, \varphi) \leq m, \quad \forall t \in[0,1], \varphi \leq 0
$$

hence by (14) and (15), we have

$$
(\Phi u)(t)=(L G u)(t) \leq L m, \quad \forall t \in[0,1], u \in(-P) .
$$

Obviously, $L m \in P$. Since $0<\xi<\lambda_{1}$, there exists $h>0$ such that

$$
0<\xi+h<\lambda_{1}
$$

By $\left(\mathrm{H}_{2}\right)$, there exists $M>0$ such that

$$
g(t, \varphi) \leq(\xi+h) \varphi+M, \quad \forall t \in[0,1], \varphi \geq 0,
$$

so by (14) and (15), we have

$$
\begin{array}{r}
(\Phi u)(t)=(L G u)(t) \leq(\xi+h)(L u)(t)+L M, \\
\forall t \in[0,1], \quad u \in P .
\end{array}
$$

Set $\widetilde{B}=(\xi+h) L$; by (37) and Lemma 9, we have $r(\widetilde{B})=$ $(\xi+h) r(L)<\lambda_{1} r(L)=1$. Equations (36) and (39) show that (8)of Lemma 6 are satisfied. Therefore, Lemma 6 guarantees that Theorem 12 is valid.

Theorem 13. Suppose that $\left(H_{1}\right)-\left(H_{4}\right)$ hold and $\xi=\rho=\gamma$. In addition, assume that

(i) $g(t, \varphi)$ is strictly increasing on $\varphi$;

(ii) there exists an even number $\tilde{n} \geq 2$ such that

$$
\lambda_{\widetilde{n}}<\gamma<\lambda_{\widetilde{n}+1}
$$

(iii) $0<\eta<\lambda_{1}$,

where $\lambda_{1}, \lambda_{\tilde{n}}$, and $\lambda_{\widetilde{n}+1}$ are defined by (10).

Then BVP (1) has at least three nontrivial solutions, containing a sign-changing solution.

Proof. By (13), we know that

$$
k(\beta, s) \geq \beta(1-\beta) s(1-s), \quad \forall s \in[0,1] .
$$
have

Since $k(t, s) \geq 0$ for any $t, s \in[0,1]$, by (12) and (41), we

$$
Z(t, s) \geq \frac{\alpha t}{1-\alpha \beta} \beta(1-\beta) s(1-s) \geq \frac{\alpha \beta(1-\beta)}{1+\alpha-\alpha \beta} t s(1-s),
$$

$\forall t, s \in[0,1]$. 
It follows from (12) that $k(\tau, s) \leq s(1-s), \forall \tau, s \in[0,1]$; so by (42) we have

$$
Z(t, s) \geq \frac{\alpha \beta(1-\beta) t}{1+\alpha-\alpha \beta} k(\tau, s), \quad \forall \tau, t, s \in[0,1] .
$$

Form (13), we easily know that

$$
\begin{aligned}
Z(t, s) & \geq \frac{\alpha t}{1-\alpha \beta} k(\beta, s) \geq \frac{\alpha t \tau}{1-\alpha \beta} k(\beta, s) \\
& \geq \frac{\alpha \beta(1-\beta) t}{1+\alpha-\alpha \beta} \frac{\alpha \tau}{1-\alpha \beta} k(\beta, s), \quad \forall t, \tau, s \in[0,1] .
\end{aligned}
$$

Therefore, by (43) and (44), we get

$$
Z(t, s) \geq \zeta t Z(\tau, s), \quad \forall \tau, t, s \in[0,1]
$$

where $\zeta=\alpha \beta(1-\beta) / 2(1+\alpha-\alpha \beta)$.

From (45) and (15), for any $u \in P \backslash\{\theta\}$, we have that

$$
\begin{aligned}
(L u)(t) & =\int_{0}^{1} Z(t, s) u(s) d s \geq \zeta t \int_{0}^{1} Z(\tau, s) u(s) d s \\
& =\zeta t(L u)(\tau), \quad \forall t \in[0,1],
\end{aligned}
$$

which means

$$
(L u)(t) \geq \zeta t\|L u\|
$$

namely,

$$
L(P \backslash\{\theta\}) \subseteq \operatorname{int} P
$$

By (48) and the condition (i), we know that $\Phi$ is strongly increasing; so condition (i) of Lemma 7 is satisfied.

By Lemma 10 we have

$$
\Phi_{P}^{\prime}=\Phi_{(-P)}^{\prime}=\gamma L, \quad \Phi_{\theta}^{\prime}=\eta L .
$$

By Lemmas 8, 9, and (49), we know that $\gamma / \lambda_{1}, \gamma / \lambda_{2}$, $\ldots, \gamma / \lambda_{n}, \ldots$ are the eigenvalues of the linear operators $\Phi_{P}^{\prime}=$ $\Phi_{(-P)}^{\prime}$. Since $\lambda_{\tilde{n}}<\gamma<\lambda_{\tilde{n}+1}$, we know that 1 is not an eigenvalue of $\Phi_{P}^{\prime}$ and $\Phi_{(-P)}^{\prime}$, and $r\left(\Phi_{P}^{\prime}\right)=r\left(\Phi_{(-P)}^{\prime}\right)=\gamma / \lambda_{1}>1$, so condition (ii) of Lemma 7 is also satisfied.

Since $g(t, 0) \equiv 0, \Phi \theta=\theta$. By (48), we know that $\Phi_{\theta}^{\prime}$ is strongly positive. By (49), Lemma 9 , and $0<\eta<\lambda_{1}$, we have $r\left(\Phi_{\theta}^{\prime}\right)=\eta / \lambda_{1}<1$. So condition (iii) of Lemma 7 is satisfied.

In the following, we prove that $\Phi_{\infty}^{\prime}=\gamma L$.

In fact, by $\left(\mathrm{H}_{2}\right)\left(\mathrm{H}_{3}\right)$ and $\xi=\rho=\gamma$, for any $\epsilon>0$, there exists $\widetilde{R}>0$ such that

$$
|g(t, \varphi)-\gamma \varphi| \leq \epsilon|\varphi|, \quad \forall t \in[0,1],|\varphi| \geq \widetilde{R} .
$$

Let $\widetilde{M}=\max _{t \in[0,1],-\widetilde{R} \leq \varphi \leq \widetilde{R}}|g(t, \varphi)|$. Then

$|(G u)(t)-\gamma u(t)|=|g(t, u(t))-\gamma u(t)| \leq \widetilde{M}+\gamma \widetilde{R}+\epsilon\|u\|$,

$$
\forall t \in[0,1], \quad\|u\| \geq \widetilde{R},
$$

and hence

$$
\|\Phi u-\gamma L u\|=\|L(G u)-\gamma L u\| \leq\|L\|(\widetilde{M}+\gamma \widetilde{R}+\epsilon\|u\|),
$$

which means

$$
\liminf _{\|u\| \rightarrow \infty} \frac{\|\Phi u-\gamma L u\|}{\|u\|} \leq \epsilon\|L\|
$$

that is,

$$
\lim _{\|u\| \rightarrow \infty} \frac{\|\Phi u-\gamma L u\|}{\|u\|}=0,
$$

so we have $\Phi_{\infty}^{\prime}=\gamma L$.

By condition (ii) and Lemma 8, we know that 1 is not the eigenvalue of $\Phi_{\infty}^{\prime}$, and the sum of the algebraic multiplicities for all eigenvalues of $\Phi_{\infty}^{\prime}$, lying in the interval $(1, \infty)$, is an even number $\tilde{n}$. So condition (iv) of Lemma 7 is satisfied. Therefore, Lemma 7 guarantees that Theorem 13 is valid.

Remark 14. In [13-15], the authors considered the boundary value problem (2). In this paper, it is obvious that we generalize and improve the nonlinear term $g$, and we also obtain that BVP (1) has at least a sign-changing solution. The methods we use are different from those of [13-15]. In [18], the author has used global bifurcation theorem to obtain signchanging solutions of the boundary value problem (3), so the methods we use are different from those of [18].

\section{Applications}

In this section, some examples are given to illustrate our main results obtained in Section 3.

Consider the following second order three-point boundary value problem:

$$
\begin{gathered}
-u^{\prime \prime}(t)=g(t, u(t)), \quad 0 \leq t \leq 1, \\
u(0)=0, \quad u(1)=\frac{1}{2} u\left(\frac{1}{2}\right) .
\end{gathered}
$$

By simple calculations, we know that $\lambda_{1} \approx 6.9497, \lambda_{2} \approx$ 39.4784, and $\lambda_{3} \approx 98.6077$ are solutions of the following equation:

$$
\sin \sqrt{x}=\frac{1}{2} \sin \frac{\sqrt{x}}{2} .
$$

Example 1. Choose

$$
\begin{aligned}
& g(t, \varphi) \\
& \quad= \begin{cases}\frac{2 \varphi+(t-8) \sqrt{\varphi},}{-26+2 t}(\varphi-1) & t \in[0,1], \varphi \in[9,+\infty), \\
+t+20, & t \in[0,1], \varphi \in(1,9), \\
20 \varphi+t \varphi^{7 / 3}, & t \in[0,1], \varphi \in[-1,1], \\
\frac{t+2}{7}(\varphi+1)-20-t, & t \in[0,1], \varphi \in(-8,-1), \\
3 \varphi+(t-1) \sqrt[3]{\varphi}, & t \in[0,1], \varphi \in(-\infty,-8] .\end{cases}
\end{aligned}
$$


Obviously, the nonlinear term $g(t, \varphi)$ can be negative when $\varphi \geq 0$ and $\xi=2, \rho=3, \eta=20$. It is easy to know that the conditions of Theorem 11 are satisfied. So BVP (55) has at least one nontrivial solution.

Example 2. Choose

$$
\begin{aligned}
& g(t, \varphi) \\
& = \begin{cases}\varphi+(t-21) \sqrt{\varphi}, & t \in[0,1], \varphi \in[144,+\infty), \\
\frac{-13+t}{11}(\varphi-1) & t \in[0,1], \varphi \in(1,144), \\
+61-t, & t \in[0,1], \varphi \in[-1,1], \\
60 \varphi+(1-t) \varphi^{7 / 3}, & \\
\frac{749+3 t^{2}+2 t}{26} & t \in[0,1], \varphi \in(-27,-1), \\
\times(\varphi+1)-61+t, & t \in[0,1], \varphi \in(-\infty,-27] . \\
30 \varphi-t+t^{2} \sqrt[3]{\varphi}, & \end{cases}
\end{aligned}
$$

It is easy to know that $\xi=1, \rho=30, \eta=60$ and the nonlinear term $g(t, \varphi)$ can be negative when $\varphi \geq 0$. The conditions of Theorem 12 are satisfied. So BVP (55) has at least one nontrivial solution.

Example 3. Choose

$$
\begin{aligned}
& g(t, \varphi) \\
& \quad= \begin{cases}\frac{60 \varphi+t \sqrt{\varphi},}{236+t} & t \in[0,1], \varphi \in[4,+\infty), \\
3 \varphi+(1+t) \varphi^{7 / 3}, & t \in[0,1], \varphi \in[-1,1], \\
\frac{t+476}{7}(\varphi+1)-4-t, & t \in[0,1], \varphi \in(-8,-1), \\
60 \varphi+t \sqrt[3]{\varphi}, & t \in[0,1], \varphi \in(-\infty,-8]\end{cases}
\end{aligned}
$$

Obviously, $\xi=\rho=\gamma=60, \eta=3$. It is easy to know that the conditions of Theorem 13 are satisfied. Thus BVP (55) has at least three nontrivial solutions, containing a sign-changing solution.

\section{Conflict of Interests}

The author declares that there is no conflict of interests regarding the publication of this paper.

\section{Acknowledgments}

The author wishes to thank the referees for their valuable suggestions. The project is supported by the National Natural Science Foundation of China (10971179), Research Award Fund for Outstanding Young Scientists of Shandong Province (BS2012SF022), and A Project of Shandong Province Higher Educational Science and Technology Program (J11LA07).

\section{References}

[1] S. P. Timoshenko, Theory of Elastic Stability, McGraw-Hill, New York, NY, USA, 1961.

[2] C. P. Gupta, "Solvability of a three-point nonlinear boundary value problem for a second order ordinary differential equation," Journal of Mathematical Analysis and Applications, vol. 168, no. 2, pp. 540-551, 1992.

[3] J. R. L. Webb, "Positive solutions of some three point boundary value problems via fixed point index theory," Nonlinear Analysis: Theory, Methods \& Applications, vol. 47, no. 7, pp. 4319-4332, 2001.

[4] G. W. Zhang and J. X. Sun, "Positive solutions of $m$-point boundary value problems," Journal of Mathematical Analysis and Applications, vol. 291, no. 2, pp. 406-418, 2004.

[5] G. W. Zhang and J. X. Sun, "Multiple positive solutions of singular second-order $m$-point boundary value problems," Journal of Mathematical Analysis and Applications, vol. 317, no. 2, pp. 442447, 2006.

[6] B. M. Liu, L. S. Liu, and Y. H. Wu, "Positive solutions for singular second order three-point boundary value problems," Nonlinear Analysis: Theory, Methods \& Applications, vol. 66, no. 12, pp. 2756-2766, 2007.

[7] R. Ma and N. Castaneda, "Existence of solutions of nonlinear $m$-point boundary-value problems," Journal of Mathematical Analysis and Applications, vol. 256, no. 2, pp. 556-567, 2001.

[8] B. M. Liu, "Positive solutions of a nonlinear three-point boundary value problem," Applied Mathematics and Computation, vol. 132, no. 1, pp. 11-28, 2002.

[9] X. Xu, "Multiplicity results for positive solutions of some semipositone three-point boundary value problems," Journal of Mathematical Analysis and Applications, vol. 291, no. 2, pp. 673689, 2004.

[10] X. Xu, D. O’Regan, and J. X. Sun, "Multiplicity results for threepoint boundary value problems with a non-well-ordered upper and lower solution condition," Mathematical and Computer Modelling, vol. 45, no. 1-2, pp. 189-200, 2007.

[11] Y. P. Guo, W. G. Ge, and S. J. Dong, “Two positive solutions for second order three point boundary value problems with sign changing nonlinearities," Acta Mathematicae Applicatae Sinica, vol. 27, no. 3, pp. 522-529, 2004.

[12] T. Bartsch and Z. Q. Wang, "On the existence of sign changing solutions for semilinear Dirichlet problems," Topological Methods in Nonlinear Analysis, vol. 7, no. 1, pp. 115-131, 1996.

[13] X. Xu and J. X. Sun, "On sign-changing solution for some threepoint boundary value problems," Nonlinear Analysis: Theory, Methods \& Applications, vol. 59, no. 4, pp. 491-505, 2004.

[14] K. Zhang and X. J. Xie, "Existence of sign-changing solutions for some asymptotically linear three-point boundary value problems," Nonlinear Analysis: Theory, Methods \& Applications, vol. 70, no. 7, pp. 2796-2805, 2009.

[15] Y. J. Cui, Y. M. Zou, and H. Y. Li, "Existence of sign-changing solutions for nonlinear operator equations and its applications," Journal of Systems Science and Mathematical Sciences, vol. 29, no. 8, pp. 1094-1101, 2009.

[16] J. X. Sun, Nonlinear Functional Analysis and Applications, Science Press, Beijing, China, 2008.

[17] B. P. Rynne, "Second-order, three-point boundary value problems with jumping non-linearities," Nonlinear Analysis: Theory, Methods \& Applications, vol. 68, no. 11, pp. 3294-3306, 2008. 
[18] B. P. Rynne, "Spectral properties and nodal solutions for second-order, $m$-point, boundary value problems," Nonlinear Analysis: Theory, Methods \& Applications, vol. 67, no. 12, pp. 3318-3327, 2007.

[19] D. L. Bai and H. F. Feng, "Eigenvalue for a singular second order three-point boundary value problem," Journal of Applied Mathematics and Computing, vol. 38, no. 1-2, pp. 443-452, 2012.

[20] Y. Z. Lin, J. Niu, and M. G. Cui, "A numerical solution to nonlinear second order three-point boundary value problems in the reproducing kernel space," Applied Mathematics and Computation, vol. 218, no. 14, pp. 7362-7368, 2012.

[21] X. Y. Liu and J. X. Sun, "Computation of topological degree of unilaterally asymptotically linear operators and its applications," Nonlinear Analysis: Theory, Methods \& Applications, vol. 71, no. 1-2, pp. 96-106, 2009.

[22] J. X. Sun and X. Y. Liu, "Computation of topological degree for nonlinear operators and applications," Nonlinear Analysis: Theory, Methods \& Applications, vol. 69, no. 11, pp. 4121-4130, 2008.

[23] J. X. Sun and X. Y. Liu, "Computation of topological degree in ordered Banach spaces with lattice structure and its application to superlinear differential equations," Journal of Mathematical Analysis and Applications, vol. 348, no. 2, pp. 927-937, 2008.

[24] H. Y. Li and F. Sun, "Existence of solutions for integral boundary value problems of second-order ordinary differential equations," Boundary Value Problems, vol. 2012, article 147, 7 pages, 2012.

[25] H. X. Lu, L. Sun, and J. X. Sun, "Existence of positive solutions to a non-positive elastic beam equation with both ends fixed," Boundary Value Problems, vol. 2012, article 56, 10 pages, 2012.

[26] D. J. Guo, Nonlinear Functional Analysis, Shandong Science and Technology Press, Jinan, China, 2nd edition, 2001. 


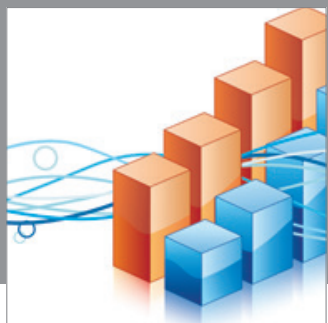

Advances in

Operations Research

mansans

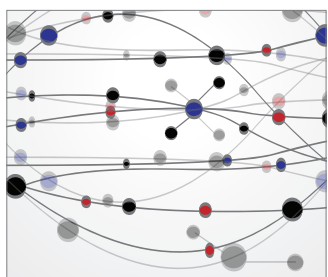

The Scientific World Journal
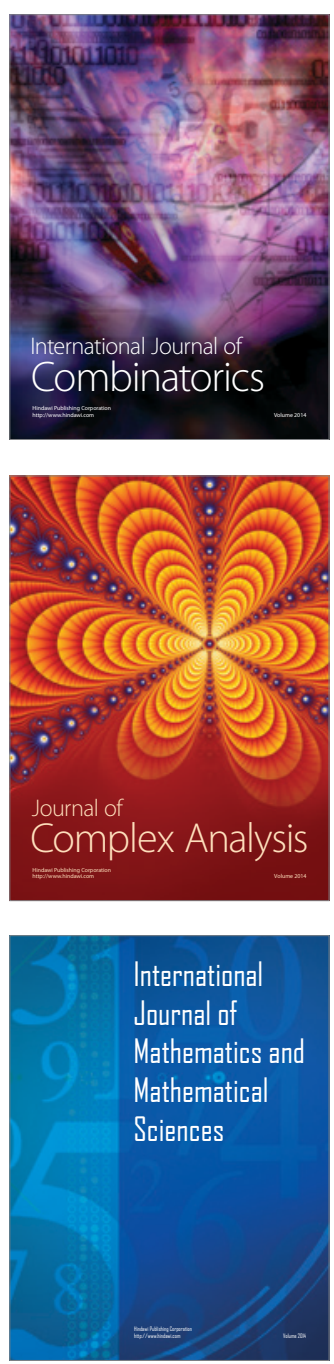
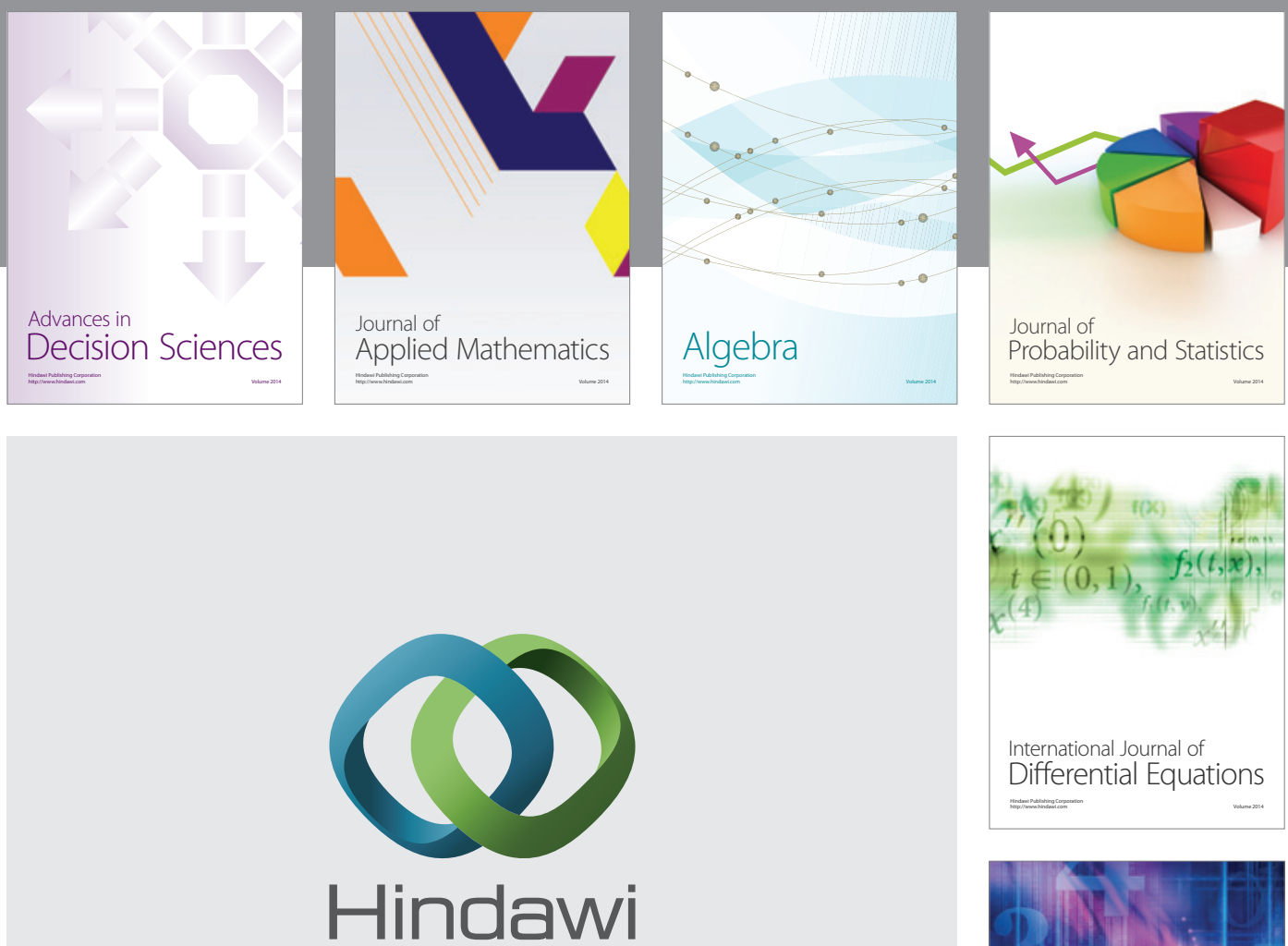

Submit your manuscripts at http://www.hindawi.com
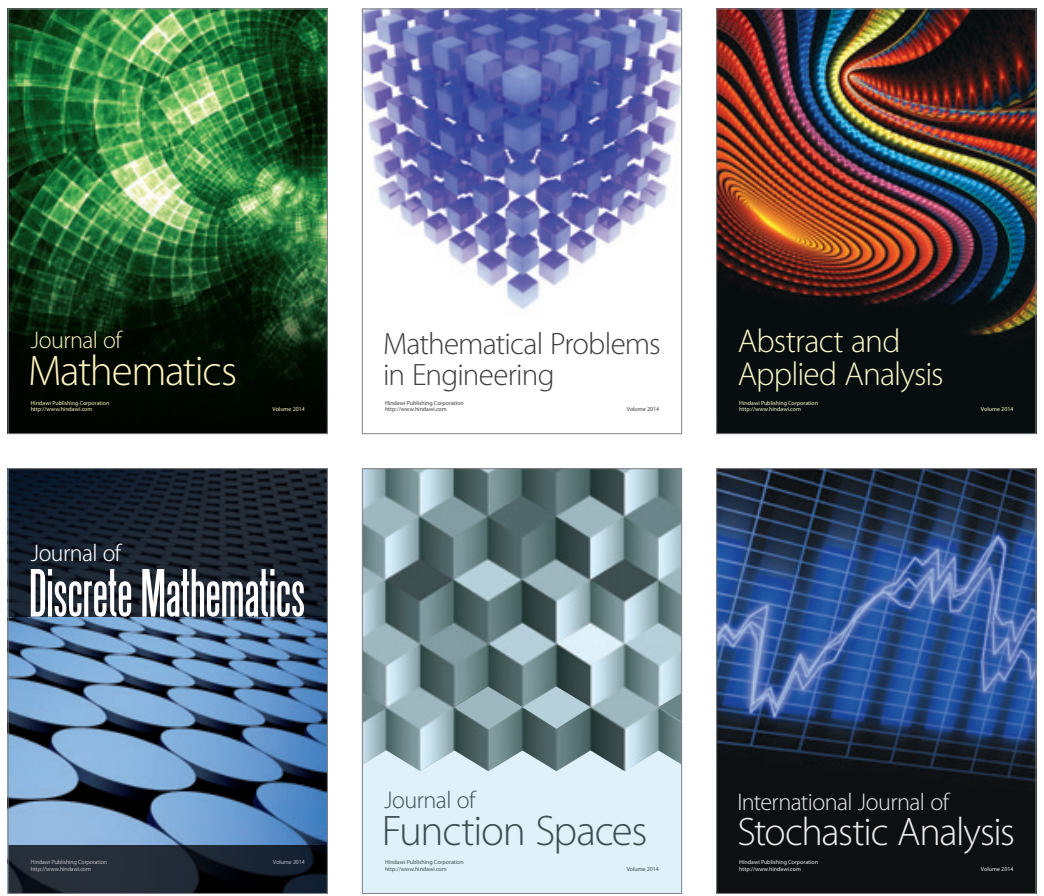

Journal of

Function Spaces

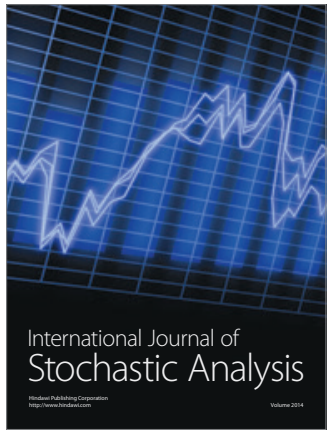

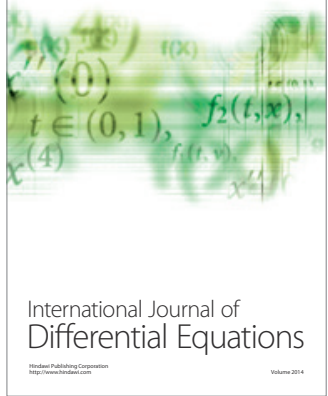
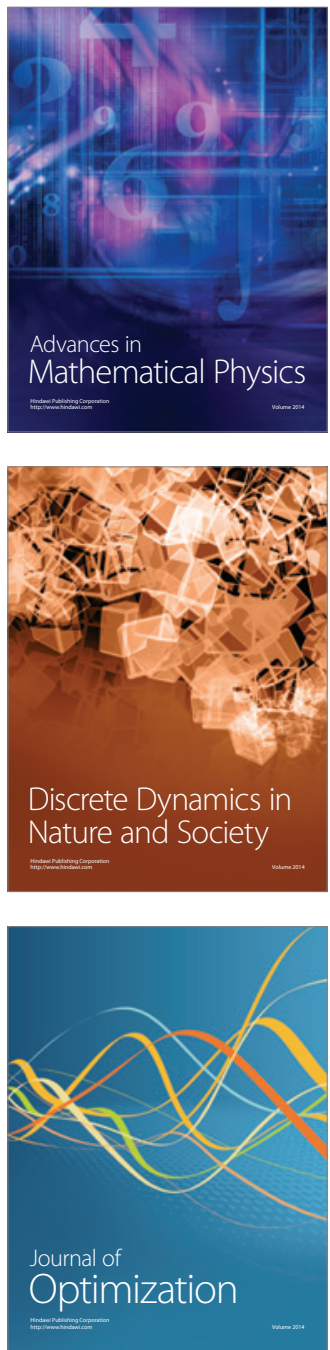